\title{
Impact of group-based dietary education on the dietary habits of female adolescents: a cluster randomized trial
}

\author{
Kazue Yamaoka ${ }^{1, *}$, Mariko Watanabe ${ }^{2}$, Eisuke $\mathrm{Hida}^{1}$ and Toshiro Tango ${ }^{1}$ \\ 'Department of Technology Assessment and Biostatistics, National Institute of Public Health, 2-3-6 Minami, \\ Wako, Saitama 351-0197, Japan: ${ }^{2}$ Department of Human Nutrition, Graduate School of Human Ecology, \\ Showa Women's University, Tokyo, Japan
}

Submitted 20 October 2009: Accepted 1 July 2010: First published online 15 September 2010

\begin{abstract}
Objective: The number of extremely thin young women has increased and education at school on maintaining an optimal weight has become important. The aim of the present study was to assess the effectiveness of a group-based homecollaborative dietary education (HCDE) programme to maintain appropriate dietary intake compared to conventional school classroom education.

Design: Two-arm cluster randomized controlled trial. Twelve classes were randomly assigned as clusters to either the HCDE group or the control group. Each participant in the HCDE group received twelve sessions of group counselling aimed at increasing energy intake at breakfast by modifying dietary intake and adopting appropriate habits. The hypothesis underlying the study was that after 6 months of HCDE the total energy intake would be increased by $627 \mathrm{~kJ}$ from baseline (primary endpoint). Secondary outcomes were differences in intake of various nutrients from baseline. Outcome measures after log transformation were examined by $t$ tests and linear mixed models (crude and baseline-adjusted).

Setting: Young women among Japanese female adolescents in Tokyo.

Subjects: Four hundred and seventy-four participants aged 13-15 years.

Results: Students in twelve classes were used for analysis ( $n$ 459). Energy intake was decreased in many of the classes during the 6-month period, especially for those in the control group. After adjustment for the baseline value, significant increases in energy intake and protein, calcium, magnesium and iron intakes at breakfast were observed $(P<0 \cdot 05)$

Conclusions: Although energy intake was increased in the HCDE group compared to the control group, further study of the HCDE is warranted.
\end{abstract}

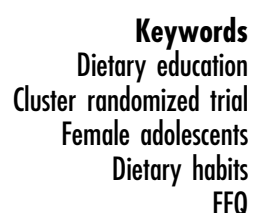

FFQ
The increase in the number of extremely thin young women is a new problem that should be addressed along with youth obesity ${ }^{(1-3)}$. These tendencies may be related to specific problems in dietary intake among young people, such as skipping breakfast and/or excessive snacking at night, etc. Nutrition is an important modifiable factor in the physical development of adolescents $^{(4)}$. Therefore, school education on appropriate nutritional intake is important.

To conduct effective dietary education, an assessment of nutritional intake is important. An FFQ is a feasible method for this purpose ${ }^{(5)}$. Recently, we developed an FFQ consisting of a list of eighty-two foods (FFQW82) ${ }^{(6)}$ for use in epidemiological studies. It includes illustrations in colour showing portion sizes. The advantage of the FFQW82 is that the dietary habits for the previous month can be analysed by meal type (breakfast, lunch and dinner) and by food groups. In particular, breakfast habits affect overall nutrient profiles ${ }^{(7,8)}$, and it is important to ascertain the breakfast habits of this age group. Using this instrument, the above-mentioned specific problems related to dietary intake among young people can be revealed and goals for dietary improvement based on scientific evidence can be planned and practised.

From the viewpoint of evidence-based nutrition, a randomized controlled trial is the most reliable scientific approach $^{(9)}$; however, in the setting of school education, randomizing individuals is not always feasible and cluster randomized trials are increasingly being utilized in the evaluation of health education interventions ${ }^{(10)}$. The aim of the present study was to assess the effectiveness of a group-based home-collaborative dietary education (HCDE), a new dietary education programme to reduce the number of extremely underweight young women, among Japanese female adolescents through a cluster randomized controlled trial. 


\section{Methods}

\section{Study design}

The study design was a cluster randomized controlled trial with before/after measurements and two intervention arms. The cluster design was necessary because randomization was performed at a school class level. The study period was 6 months.

\section{Participants}

Study participants were female adolescents, aged 13-15 years, who resided in a metropolitan area of Tokyo, Japan. They were recruited primarily through the explanation of the survey while being accompanied by a parent or guardian at school in September 2007. All the participants (students and their guardians) received a verbal description of the study and were asked to read and sign an informed consent form. Upon providing informed consent, all participants completed the FFQW82. The study protocol was approved by the Ethics Committee of the Showa Women's University in Tokyo, Japan. Those who refused were excluded from the study.

\section{Randomization}

School classes were randomly assigned to either the HCDE or control group, with the use of a randomization list (random permutated blocks with block size 2, stratified by grade) in September 2007.

\section{Intervention}

The HCDE was provided to all individuals in the classes assigned to the HCDE group in addition to conventional health science education. The HCDE group received twelve sessions of group counselling, with some counselling given individually after the group counselling. They were asked to put into practice at home what they had learned at school with the assistance of their parents.

We developed the HCDE based on some of the strategies described in previous studies ${ }^{(1,4)}$. The purposes of the HCDE were to give an adolescent female scientific information, both in a group setting and occasionally in an individual setting, about her actual physical and dietary condition, to raise her motivation to correct deficiencies and to help her to recognize on her own the need for behavioural modification. Nutritional education provided scientific information on the body and diet; appropriate energy intake for breakfast and other meals using FFQW82 ${ }^{(6)}$; foods to include in modifying the diet and basic cooking; maintaining appropriate body weight and prevention of lifestylerelated disease, etc. The first session of group counselling was held 2 weeks after the baseline FFQW82 and subsequent sessions were held at 2-week intervals. Dietary advice was given by a schoolteacher who was also a dietitian. The advice was tailored to each participant on the basis of the results of the FFQW82, with a booklet illustrating the ideal meal pattern corresponding to the needed energy intake, which was calculated based on information relative to height, age and physical activity level, which were determined for each student. Furthermore, to optimize total energy intake in a day, the HCDE aimed to teach students to equalize energy intake among the three meals (breakfast, lunch and dinner), to keep protein energy intake around $15-20 \%$, fat energy intake around $20-30 \%$ and carbohydrate energy intake around 50-70\% of total energy. In addition, the HCDE was devised to optimize the intake of whole-grain products, vegetables and fruit, low-fat milk, beans, fish, meat and eggs. The students were asked to discuss the information gained from the HCDE with their mother or guardian. A liaison notebook was used for communication among the student, her guardian (mainly mother) and the dietitian with regard to the education. The student, guardian and dietitian could all make notes in this notebook. The student described what was learned at the group counselling session and what she put into practice at home, the parent or guardian entered his/her impressions and the dietitian commented on the issues raised. This created a shared understanding among the three entities. As each class was heterogeneous in terms of body weight, it was considered that some classes might include overweight individuals, although they were expected to be fewer than underweight adolescents. In such cases, the dietitian offered individual counselling on weight reduction.

The control group was given only conventional health science education in their school classes. In addition, they were provided with general written information about diet and the results of FFQW82, but no detailed explanation was provided. After the intervention period, all control group participants were provided with the HCDE so that they would not be deprived of the information needed for good health and nutrition.

Assessments using the FFQW82 were made at baseline and again at 6 months between September 2007 and March 2008, depending upon when the baseline assessment was made, to evaluate whether there had been a change in dietary intake.

\section{Study bypotbesis}

The hypothesis underlying the study was that participation in the HCDE might increase total energy intake by $627 \mathrm{~kJ}(150 \mathrm{kcal})$ from baseline (primary endpoint) in thin girls after 6 months of education whereas such an increase would not occur in the control group. The estimated energy requirement (EER) for adolescents of this age was recommended by the Japan Ministry of Health, Labour and Welfare to be $8569 \mathrm{~kJ}$ even for those with a low level of physical activity. On the other hand, through our experience with an previous survey ${ }^{(11)}$, we found that the average energy intake of persons of this age was around 7106 (SD 836) kJ. We decided on an increase of $627 \mathrm{~kJ}$ as the tentative recommendation for our participants. The amount of $627 \mathrm{~kJ}$ corresponds to one rice ball ('Onigiri'), a popular food in Japan, or a cup of milk. 
We considered encouraging the students to add such foods to breakfast because compliance with this recommendation would be most easy at that time.

\section{Outcome}

The primary endpoint was an increase in total energy intake of $627 \mathrm{~kJ}$ from baseline after 6 months of education. The difference in the primary endpoints of the HCDE and control groups was the effect size of the present study. Secondary outcomes were determined based on differences in intake of various nutrients from baseline.

\section{Sample size}

The sample size needed for the study was determined based on the information on the effect size with a significance level of $5 \%$ (two-sided), a power of $80 \%$, equal allocation, a unit of cluster of thirty-seven students and an intra-class correlation of $0 \cdot 02$.

\section{Statistical analyses}

Outcome measures after log transformation were examined. Mean and SD values by classes of outcome measures in the HCDE and control groups were compared using Student's $t$ test. This assumed no within variance; therefore, we added the following analyses: namely, to assess within-person $\left(S_{w}^{2}\right)$ and between-person $\left(S_{b}^{2}\right)$ intra-class variances, linear mixed models were used, which included a crude model (model 1) and a baseline-adjusted model (model 2) using individual data ${ }^{(12)}$.

As the participants who dropped out before the end of the 6-month intervention period were not included in the analysis, an 'intent-to-treat' (ITT) approach was performed by the Last Observation Carried Forward method.

All tests of significance were two-sided with a $5 \%$ significance level. All statistical analyses were performed using the SAS statistical software package version $9 \cdot 13$ for Windows (SAS Institute Inc., Cary, NC, USA).

\section{Results}

Figure 1 is a diagram showing the flow of clusters and participants from enrolment to analysis $\left(\mathrm{CONSORT}^{(13)}\right)$. We randomly assigned twelve classes as clusters ( $n 466$ students)

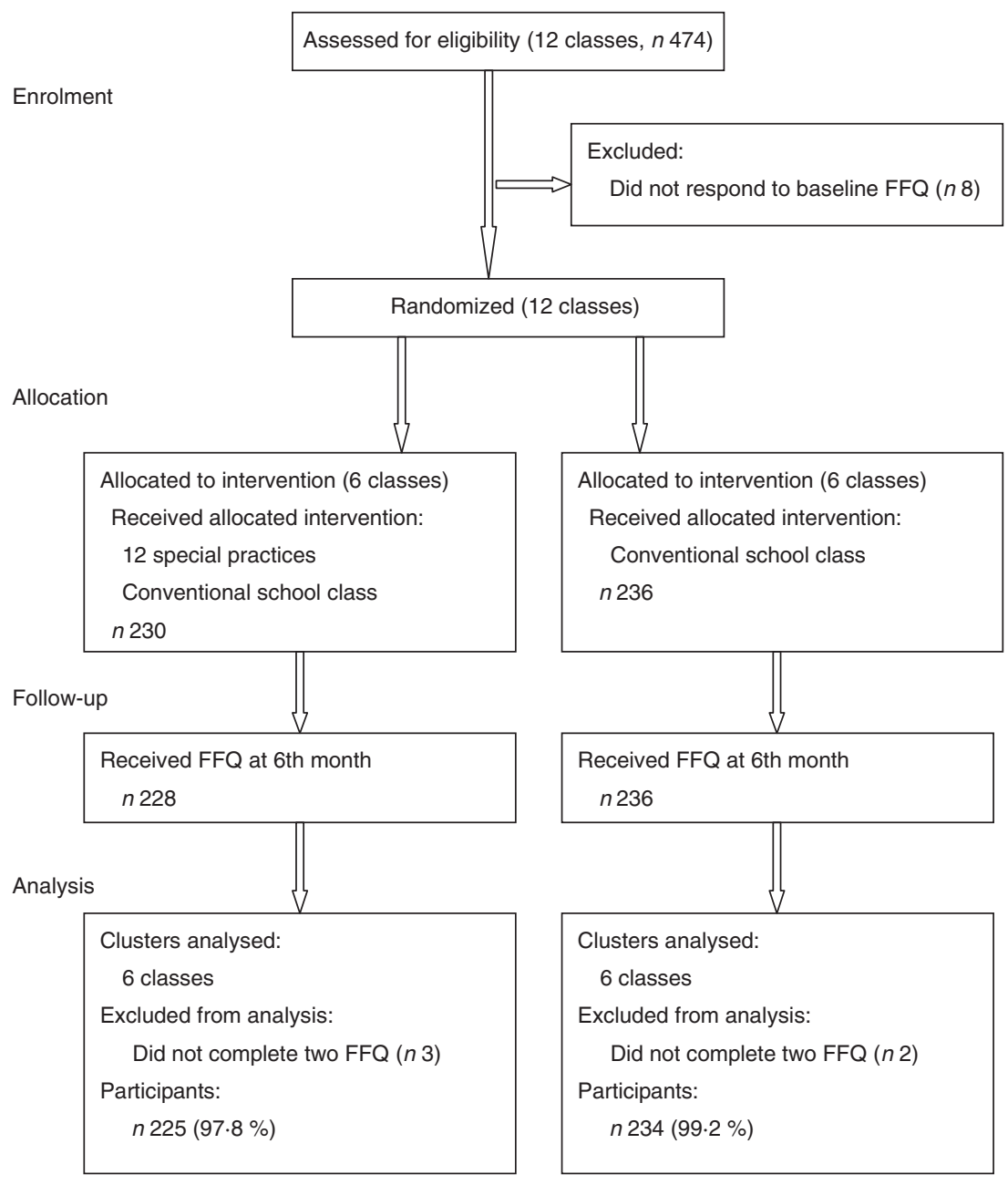

Fig. 1 Diagram showing flow of clusters and participants through entire study period (CONSORT) 
to either the HCDE group (six classes, $n 230$ students) or the control group (six classes, $n 236$ students). Eligible for analysis were 459 students $(\mathrm{HCDE}=225$, control $=234)$. Table 1 shows the average nutrient intake estimated by FFQW82, physical measurements, and age of each group at baseline. Seven in the HCDE group and six in the

Table 1 Basic statistics at baseline on nutrient intakes estimated by FFQW82, physical measurements and age

\begin{tabular}{|c|c|c|c|c|}
\hline & \multicolumn{2}{|c|}{ HCDE group } & \multicolumn{2}{|c|}{ Control group } \\
\hline & Mean & SD & Mean & SD \\
\hline \multicolumn{5}{|c|}{ Energy (kcal)† } \\
\hline Breakfast & $352 \cdot 1$ & $61 \cdot 4$ & $351 \cdot 7$ & $60 \cdot 4$ \\
\hline Lunch & $511 \cdot 5$ & 95.9 & $509 \cdot 1$ & $89 \cdot 3$ \\
\hline Dinner & $682 \cdot 0$ & $59 \cdot 8$ & $693 \cdot 7$ & $58 \cdot 1$ \\
\hline $1 \mathrm{~d}$ & $1545 \cdot 5$ & $133 \cdot 1$ & $1553 \cdot 5$ & $120 \cdot 8$ \\
\hline \multicolumn{5}{|l|}{ Protein (g) } \\
\hline Breakfast & $12 \cdot 9$ & $3 \cdot 7$ & $12 \cdot 8$ & $3 \cdot 5$ \\
\hline Lunch & $15 \cdot 9$ & $3 \cdot 9$ & $15 \cdot 6$ & $3 \cdot 4$ \\
\hline Dinner & $28 \cdot 9$ & $3 \cdot 1$ & $29 \cdot 2$ & $2 \cdot 9$ \\
\hline $1 \mathrm{~d}$ & $57 \cdot 1$ & $6 \cdot 7$ & $57 \cdot 1$ & $6 \cdot 0$ \\
\hline \multicolumn{5}{|l|}{ Fat $(\mathrm{g})$} \\
\hline Breakfast & $11 \cdot 4$ & $4 \cdot 3$ & $11 \cdot 5$ & $4 \cdot 3$ \\
\hline Lunch & $15 \cdot 1$ & $4 \cdot 1$ & $14 \cdot 9$ & $3 \cdot 8$ \\
\hline Dinner & $23 \cdot 8$ & $2 \cdot 7$ & $24 \cdot 1$ & $2 \cdot 7$ \\
\hline $1 \mathrm{~d}$ & $50 \cdot 5$ & $8 \cdot 4$ & $50 \cdot 8$ & $8 \cdot 2$ \\
\hline \multicolumn{5}{|c|}{ Carbohydrate (g) } \\
\hline Breakfast & $49 \cdot 8$ & $9 \cdot 0$ & $49 \cdot 1$ & $6 \cdot 8$ \\
\hline Lunch & $69 \cdot 0$ & $11 \cdot 1$ & $69 \cdot 1$ & $10 \cdot 6$ \\
\hline Dinner & $81 \cdot 5$ & $6 \cdot 7$ & $83 \cdot 1$ & $6 \cdot 4$ \\
\hline $1 \mathrm{~d}$ & $200 \cdot 2$ & $15 \cdot 7$ & $201 \cdot 4$ & $13 \cdot 3$ \\
\hline \multicolumn{5}{|c|}{ Potassium (mg) } \\
\hline Breakfast & $246 \cdot 4$ & $72 \cdot 0$ & $232 \cdot 3$ & $32 \cdot 4$ \\
\hline Lunch & $99 \cdot 4$ & $129 \cdot 2$ & $79 \cdot 5$ & $54 \cdot 7$ \\
\hline Dinner & $221 \cdot 3$ & $169 \cdot 6$ & $191 \cdot 9$ & $60 \cdot 6$ \\
\hline $1 \mathrm{~d}$ & $714 \cdot 5$ & $346 \cdot 7$ & $669 \cdot 0$ & $156 \cdot 2$ \\
\hline \multicolumn{5}{|l|}{ Calcium (mg) } \\
\hline Breakfast & $133 \cdot 2$ & $71 \cdot 6$ & $134 \cdot 1$ & $67 \cdot 6$ \\
\hline Lunch & $66 \cdot 2$ & $26 \cdot 6$ & $63 \cdot 8$ & $19 \cdot 1$ \\
\hline Dinner & $133 \cdot 6$ & $32 \cdot 6$ & $137 \cdot 4$ & $30 \cdot 8$ \\
\hline $1 \mathrm{~d}$ & $351 \cdot 9$ & $117 \cdot 3$ & $358 \cdot 0$ & $105 \cdot 6$ \\
\hline \multicolumn{5}{|c|}{ Magnesium (mg) } \\
\hline Breakfast & $40 \cdot 3$ & $13 \cdot 0$ & $40 \cdot 2$ & $11 \cdot 7$ \\
\hline Lunch & $39 \cdot 8$ & $11 \cdot 5$ & $39 \cdot 1$ & 8.9 \\
\hline Dinner & $86 \cdot 2$ & $13 \cdot 0$ & $87 \cdot 4$ & $12 \cdot 1$ \\
\hline $1 d$ & $167 \cdot 3$ & $27 \cdot 2$ & $167 \cdot 8$ & $23 \cdot 4$ \\
\hline \multicolumn{5}{|l|}{ Iron (mg) } \\
\hline Breakfast & $1 \cdot 6$ & $0 \cdot 6$ & $1 \cdot 6$ & 0.5 \\
\hline Lunch & $2 \cdot 1$ & 0.5 & $2 \cdot 1$ & 0.5 \\
\hline Dinner & $3 \cdot 5$ & $0 \cdot 6$ & $3 \cdot 6$ & 0.5 \\
\hline $1 \mathrm{~d}$ & $6 \cdot 2$ & $1 \cdot 0$ & $6 \cdot 2$ & 0.9 \\
\hline \multicolumn{5}{|c|}{ Dietary fibre (g) } \\
\hline Breakfast & $2 \cdot 7$ & $0 \cdot 7$ & $2 \cdot 7$ & $0 \cdot 7$ \\
\hline Lunch & $3 \cdot 1$ & $0 \cdot 7$ & $3 \cdot 1$ & $0 \cdot 6$ \\
\hline Dinner & $5 \cdot 3$ & $0 \cdot 7$ & $5 \cdot 4$ & $0 \cdot 7$ \\
\hline $1 d$ & $9 \cdot 8$ & $1 \cdot 4$ & $9 \cdot 8$ & $1 \cdot 2$ \\
\hline \multicolumn{5}{|l|}{ Salt (g) } \\
\hline Breakfast & $2 \cdot 1$ & $0 \cdot 6$ & $2 \cdot 1$ & 0.6 \\
\hline Lunch & $2 \cdot 1$ & $0 \cdot 8$ & $2 \cdot 0$ & $0 \cdot 8$ \\
\hline Dinner & $4 \cdot 3$ & 0.9 & $4 \cdot 3$ & $0 \cdot 8$ \\
\hline $1 d$ & $7 \cdot 6$ & $1 \cdot 4$ & $7 \cdot 6$ & $1 \cdot 2$ \\
\hline Height (m) & $1 \cdot 54$ & 0.06 & 1.54 & 0.06 \\
\hline Weight (kg) & $44 \cdot 8$ & $8 \cdot 1$ & $45 \cdot 0$ & $7 \cdot 9$ \\
\hline $\mathrm{BMI}\left(\mathrm{kg} / \mathrm{m}^{2}\right)$ & $18 \cdot 9$ & $2 \cdot 7$ & $19 \cdot 0$ & $2 \cdot 8$ \\
\hline Age (years) & $13 \cdot 9$ & $0 \cdot 7$ & $14 \cdot 0$ & 0.6 \\
\hline
\end{tabular}

FFQW82, FFQ with eighty-two food items; HCDE, home-collaborative dietary education.

$+1 \mathrm{kcal}=4 \cdot 184 \mathrm{~kJ}$. control group were overweight $\left(\mathrm{BMI} \geq 25 \mathrm{~kg} / \mathrm{m}^{2}\right)$. There were considerably more underweight adolescents ( $48 \%$ for $\mathrm{BMI} \leq 18 \cdot 5 \mathrm{~kg} / \mathrm{m}^{2}, 30 \%$ for age-adjusted $\mathrm{BMI}^{(14)}$ ) than overweight adolescents ( $3 \%$ for $\mathrm{BMI} \geq 25 \mathrm{~kg} / \mathrm{m}^{2}, 14 \%$ for age-adjusted $\mathrm{BMI}^{(15)}$ ) in our study groups. The Japan Ministry of Health, Labour and Welfare has issued an alert over the problem of the increasing number of underweight adolescents as well as the problem of youth overweight. The most important concern was to reduce the number of underweight adolescents. Therefore, to increase the average energy intake was the primary concern in evaluating the effects of the dietary education although the population in each class was heterogeneous. Figure 2 shows the average energy intake of each class at baseline and at 6 months. Data were logarithmically transformed to achieve normal distributions. In the case of no intake ( 0 value), we added 1 as a small value. Dotted lines denote the mean value of the classes in the HCDE group and solid lines denote those of the control group. Energy intake tended to decrease during the 6 months, especially in the control group.

Table 2 shows the mean values (log-transformed) of the difference from baseline to 6 months after beginning the education between the HCDE and control groups, the results of Student's $t$ test for the differences in these values between the HCDE and control groups, and the results of the linear mixed models (models 1 and 2). When adjusting for the baseline value, model 2 showed a significant increase in energy, protein, calcium, magnesium and iron intakes at breakfast and in calcium intake for a total of $1 \mathrm{~d}$ in the HCDE group compared to the control group $(P<0.05$ for all). In addition, the analysis excluding the obese adolescents at baseline produced a similar result. The difference in BMI from baseline to 6 months after the beginning of the education between the HCDE and the control groups was not significant. As for the ITT analysis, except for protein and iron, a similar result was observed (not shown here).

\section{Discussion}

The present study assessed the effectiveness of the HCDE to reduce the number of extremely thin young women among Japanese female adolescents through a cluster randomized controlled trial with 459 female adolescents as participants. Many of the participants tended to decrease energy intake during the 6-month study period, especially in the control group. After adjustment for the baseline value, energy intake and intakes of protein, calcium, magnesium and iron at breakfast increased significantly in the HCDE group compared to the control group. An HCDE that is focused on promoting increased dietary intake at breakfast is likely to improve energy intake in female adolescents. 

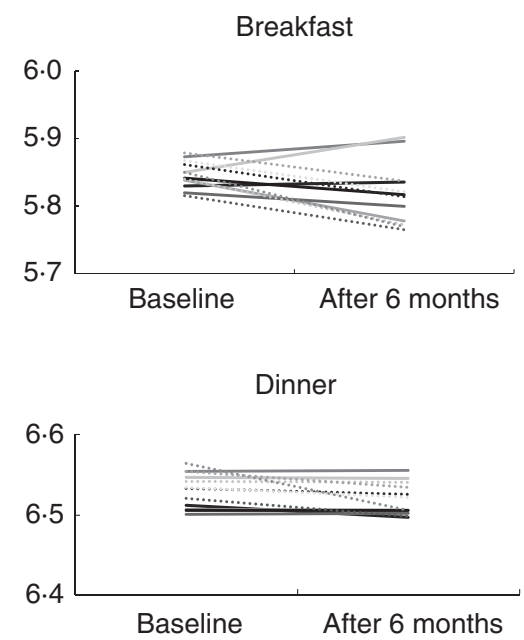
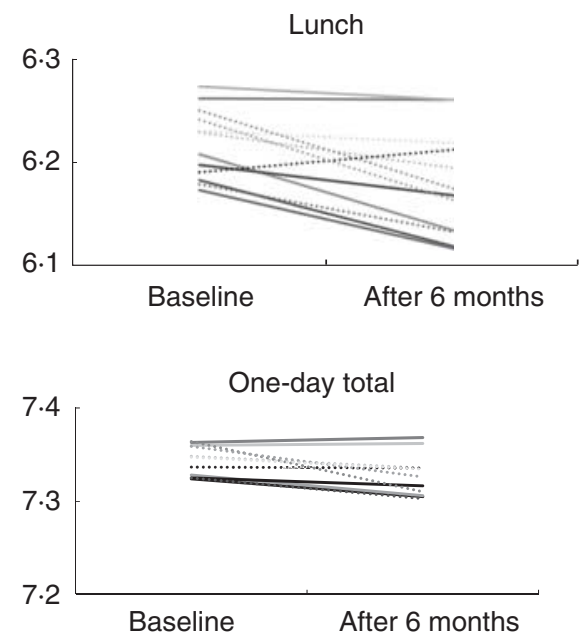

Fig. 2 Average energy intake (log-transformed) at baseline and at 6 months according to class. Solid line denotes mean value for home-collaborative dietary education group classes and dotted line denotes mean value for control group classes

\section{The bome-collaborative dietary education}

Adolescents, particularly young women, feel tremendous pressure to be thin and may try to achieve this goal through poor and sometimes dangerous nutritional choices. In Japan, the number of thin female adolescents has increased in the past few decades ${ }^{(2,16)}$ and poor nutrition results in both present and future poor health ${ }^{(1)}$. Nutrition education aimed at motivating adolescents to adopt good eating habits is very important. In conducting effective nutrition education, it is essential to make participants realize and accept responsibility for their nutritional condition and problems and the relationship of these to their future health. It is also relevant to incorporate scientific evidence that supports good nutrition into nutrition education. Although a tendency for decreased intake among the participants was observed in the present study, in the HCDE group nutritional intake was maintained despite the general situation of declining nutritional intake in this age group. In particular, there was clearly an improvement in nutritional intake at breakfast. Ask et $a l^{(17)}$ showed that serving breakfast changed the dietary intake to a more healthy profile in 15-year-old adolescents. In our study, an effect for breakfast was observed in the HCDE group in comparison with the control group, who received only conventional education. But for dinner, only a weak tendency, without significance, was observed for increased intake of many nutrients in the HCDE group in comparison with the control group. Family meals ${ }^{(18)}$ and mothers' concern for healthy eating ${ }^{(19)}$ are associated with a higher-quality diet among adolescents. In the present study, we encouraged the student's mother or guardian to help her to improve dietary intake. The implication is that effectiveness of the home-collaborative nutrition education method has been shown with regard to nutrition at breakfast and dinner.

\section{Advantages and limitations of the study}

To examine the effectiveness of a nutrition education programme by a cluster randomized clinical trial is useful in school-age students. School education is useful as a population approach. Although the review by Doak et $a l .{ }^{(3)}$ was concerned with programmes to prevent overweight among adolescents, which differed from our study on increasing weight among underweight adolescents, the effectiveness of a school-based programme was shown. A key property of cluster randomization trials is that inferences are frequently intended to apply at the individual level, whereas randomization is at the cluster or group level. The present results indicated that homecollaborative nutrition education in a school programme is effective in improving nutritional intake.

The present study had some limitations. First, the participants were limited to female adolescents of 13-15 years of age, living in an urban area. Thus, we cannot discuss or draw conclusions on the dietary habits of male adolescents or on adolescents in rural areas. There is need for additional work in the future that includes these groups. Second, in school education, we usually cannot give personal counselling to all of the students. Considering this situation, we have performed a cluster randomization trial. It was not unexpected that there were considerably more underweight than overweight adolescents, considering that the present study was carried out in Japan. The most important concern was to reduce the number of underweight adolescents. Therefore, to increase the average energy intake was the primary concern in evaluating the effects of dietary education although the population in each class was heterogeneous. We responded to the heterogeneity by including personal counselling in addition to group counselling for both overweight and underweight students. The heterogeneity of the study population might 
Table 2 Mean and SD of the difference from baseline in each group and the difference between groups by nutrient: log-transformed data

\begin{tabular}{|c|c|c|c|c|c|c|c|c|c|c|c|c|}
\hline & \multicolumn{2}{|c|}{ HCDE group } & \multicolumn{2}{|c|}{ Control group } & \multicolumn{2}{|c|}{ Difference } & \multicolumn{2}{|c|}{ Model 1} & \multicolumn{4}{|c|}{ Model 2} \\
\hline & Mean & SD & Mean & SD & Mean & SD & $\beta_{1}$ & $P$ value* & $\beta_{1}$ & $P$ value* & $\beta_{2}{ }^{\dagger}$ & $d f \ddagger$ \\
\hline \multicolumn{13}{|c|}{ Energy (kcal)§ } \\
\hline Breakfast & -0.011 & 0.035 & -0.055 & 0.019 & 0.044 & 0.028 & -0.044 & 0.026 & -0.043 & 0.033 & -0.285 & 443 \\
\hline Lunch & -0.040 & 0.031 & -0.034 & 0.037 & -0.006 & 0.034 & 0.006 & 0.763 & -0.014 & 0.133 & -0.320 & 446 \\
\hline Dinner & -0.001 & 0.008 & -0.021 & 0.020 & 0.020 & 0.015 & -0.020 & 0.047 & 0.011 & 0.284 & -0.556 & 434 \\
\hline $1 d$ & -0.011 & 0.009 & -0.021 & 0.017 & 0.010 & 0.014 & -0.010 & 0.234 & -0.008 & 0.386 & $-0 \cdot 318$ & 446 \\
\hline \multicolumn{13}{|l|}{ Protein } \\
\hline Breakfast & -0.031 & 0.038 & -0.085 & 0.029 & 0.053 & 0.034 & -0.052 & 0.052 & -0.052 & 0.045 & -0.253 & 443 \\
\hline Lunch & -0.039 & 0.015 & -0.021 & 0.029 & -0.019 & 0.023 & 0.019 & 0.296 & 0.016 & 0.359 & -0.350 & 445 \\
\hline Dinner & -0.006 & 0.010 & -0.026 & 0.023 & 0.020 & 0.018 & -0.020 & 0.081 & -0.016 & $0 \cdot 113$ & -0.359 & 446 \\
\hline $1 d$ & -0.015 & 0.005 & -0.028 & 0.020 & 0.012 & 0.015 & -0.012 & 0.202 & -0.011 & 0.231 & -0.288 & 446 \\
\hline \multicolumn{13}{|l|}{ Fat } \\
\hline Breakfast & -0.054 & 0.057 & -0.123 & 0.041 & 0.069 & 0.050 & -0.067 & 0.077 & -0.065 & 0.083 & -0.288 & 443 \\
\hline Lunch & -0.069 & 0.028 & -0.035 & 0.051 & -0.034 & 0.041 & 0.034 & $0 \cdot 188$ & 0.031 & 0.251 & -0.385 & 446 \\
\hline Dinner & -0.004 & 0.018 & -0.015 & 0.021 & 0.012 & 0.019 & -0.011 & 0.338 & -0.007 & 0.535 & -0.364 & 446 \\
\hline $1 \mathrm{~d}$ & -0.025 & 0.018 & -0.036 & 0.031 & 0.011 & 0.025 & -0.011 & 0.486 & -0.008 & 0.624 & -0.320 & 446 \\
\hline \multicolumn{13}{|l|}{ Carbohydrate } \\
\hline Breakfast & -0.016 & 0.020 & -0.042 & 0.024 & 0.026 & 0.022 & -0.027 & 0.137 & -0.030 & 0.076 & -0.439 & 443 \\
\hline Lunch & -0.034 & 0.039 & -0.030 & 0.030 & -0.004 & 0.035 & 0.004 & 0.857 & 0.007 & 0.781 & -0.507 & 445 \\
\hline Dinner & 0.001 & 0.007 & -0.016 & 0.021 & 0.017 & 0.016 & -0.017 & 0.087 & -0.010 & 0.313 & $-0 \cdot 370$ & 446 \\
\hline $1 d$ & -0.009 & 0.012 & -0.017 & 0.017 & 0.008 & 0.015 & -0.008 & 0.380 & -0.005 & 0.602 & -0.380 & 446 \\
\hline \multicolumn{13}{|l|}{ Potassium } \\
\hline Breakfast & -0.031 & 0.083 & 0.024 & 0.013 & -0.055 & 0.060 & 0.055 & $0 \cdot 136$ & 0.022 & $0 \cdot 138$ & $-0 \cdot 806$ & 443 \\
\hline Lunch & -0.046 & $0 \cdot 169$ & 0.026 & 0.012 & -0.072 & $0 \cdot 120$ & 0.071 & 0.319 & 0.003 & 0.922 & $-0 \cdot 860$ & 445 \\
\hline Dinner & -0.069 & $0 \cdot 131$ & -0.010 & 0.018 & -0.059 & 0.093 & 0.059 & 0.297 & -0.001 & 0.432 & -0.997 & 446 \\
\hline $1 \mathrm{~d}$ & -0.066 & 0.085 & -0.054 & 0.034 & -0.012 & 0.065 & 0.012 & 0.753 & -0.005 & 0.704 & -0.668 & 446 \\
\hline \multicolumn{13}{|l|}{ Calcium } \\
\hline Breakfast & -0.050 & 0.088 & -0.229 & 0.068 & 0.179 & 0.079 & -0.178 & 0.005 & -0.172 & 0.005 & -0.230 & 443 \\
\hline Lunch & -0.096 & 0.037 & -0.053 & 0.043 & -0.043 & 0.040 & 0.042 & $0 \cdot 126$ & 0.036 & $0 \cdot 149$ & -0.384 & 445 \\
\hline Dinner & -0.003 & 0.029 & -0.047 & 0.046 & 0.044 & 0.039 & -0.044 & 0.077 & -0.032 & $0 \cdot 130$ & -0.337 & 446 \\
\hline $1 \mathrm{~d}$ & -0.032 & 0.023 & -0.089 & 0.050 & 0.057 & 0.039 & -0.057 & 0.041 & -0.049 & 0.058 & -0.254 & 446 \\
\hline \multicolumn{13}{|l|}{ Magnesium } \\
\hline Breakfast & -0.025 & 0.056 & -0.099 & 0.037 & 0.074 & 0.047 & -0.074 & 0.021 & -0.071 & 0.031 & -0.256 & 442 \\
\hline Lunch & -0.047 & 0.022 & -0.042 & 0.034 & -0.005 & 0.029 & 0.005 & 0.812 & -0.004 & 0.860 & -0.489 & 432 \\
\hline Dinner & -0.006 & 0.013 & -0.042 & 0.038 & 0.036 & 0.028 & -0.357 & 0.054 & -0.030 & 0.015 & -0.333 & 446 \\
\hline $1 d$ & -0.018 & 0.015 & -0.044 & 0.031 & 0.027 & 0.025 & -0.027 & 0.090 & -0.025 & 0.091 & -0.277 & 446 \\
\hline \multicolumn{13}{|l|}{ Iron } \\
\hline Breakfast & $4 \cdot 888$ & 0.025 & $4 \cdot 844$ & 0.014 & 0.044 & 0.020 & -0.044 & 0.043 & -0.043 & 0.031 & -0.420 & 443 \\
\hline Lunch & $5 \cdot 049$ & 0.036 & $5 \cdot 059$ & 0.030 & -0.009 & 0.033 & 0.009 & 0.640 & 0.008 & 0.716 & -0.297 & 446 \\
\hline Dinner & $5 \cdot 019$ & 0.008 & 5.004 & 0.021 & 0.016 & 0.016 & -0.016 & $0 \cdot 120$ & -0.008 & 0.397 & -0.569 & 446 \\
\hline $1 d$ & $5 \cdot 366$ & 0.008 & $5 \cdot 356$ & 0.016 & 0.009 & 0.013 & -0.010 & 0.319 & -0.006 & 0.479 & -0.573 & 446 \\
\hline \multicolumn{13}{|l|}{ Fibre } \\
\hline Breakfast & -0.011 & 0.036 & -0.040 & 0.032 & 0.028 & 0.034 & -0.028 & $0 \cdot 185$ & -0.027 & 0.269 & -0.292 & 441 \\
\hline Lunch & -0.018 & 0.016 & -0.023 & 0.023 & 0.005 & 0.020 & -0.005 & 0.737 & -0.004 & 0.817 & -0.413 & 445 \\
\hline Dinner & -0.001 & 0.012 & -0.027 & 0.029 & 0.026 & 0.023 & -0.026 & 0.076 & -0.020 & 0.145 & -0.383 & 446 \\
\hline $1 d$ & -0.010 & 0.012 & -0.028 & 0.026 & 0.017 & 0.021 & -0.017 & $0 \cdot 178$ & -0.015 & 0.297 & -0.373 & 446 \\
\hline \multicolumn{13}{|l|}{ Salt } \\
\hline Breakfast & -0.007 & 0.030 & -0.038 & 0.029 & 0.031 & 0.030 & -0.031 & $0 \cdot 101$ & -0.029 & 0.153 & -0.271 & 442 \\
\hline Lunch & -0.026 & 0.018 & -0.020 & 0.035 & -0.006 & 0.028 & 0.006 & 0.770 & 0.001 & 0.959 & -0.333 & 445 \\
\hline Dinner & -0.002 & 0.014 & -0.037 & 0.037 & 0.035 & 0.028 & -0.035 & 0.058 & -0.031 & 0.096 & -0.299 & 446 \\
\hline $1 \mathrm{~d}$ & -0.008 & 0.015 & -0.031 & 0.026 & 0.024 & 0.022 & -0.024 & 0.092 & -0.022 & 0.130 & -0.266 & 446 \\
\hline
\end{tabular}

HCDE, home-collaborative dietary education.

Data were log-transformed.

Model 1: mixed model.

Model 2: mixed model with baseline adjustment.

${ }^{\star} P$ value $<0.0001$ for all.

$+\beta_{2}$ : effect of baseline value.

$\ddagger d$ for $\beta_{1}=10$.

$\S 1 \mathrm{kcal}=4 \cdot 184 \mathrm{~kJ}$.

have lessened the overall effects of the study; however, we consider that this is a feasible approach to assess school dietary education. Third, we could not observe a significant effect on BMI. As the present study aimed to examine the effect of HCDE on change in dietary habits, the number of participants was not sufficient to provide power to examine an effect. Furthermore, the intervention period of 6 months may not be long enough to determine whether the education had an effect on the health status of the adolescents. 


\section{Conclusion}

In conclusion, although the HCDE appears to have possibilities as a means of improving energy intake in female adolescents against the background of a general situation of decreased nutrition in this age group, further study on the application of the HCDE is warranted.

\section{Acknowledgements}

The present study was financially supported by the Ministry of Education, Culture, Sports, Science and Technology in Japan Grant-in-Aid for Scientific Research Grant C in 2007-2008 (Grant no. 19500693) and 2009 (Grant no. 20175128). The corresponding author confirmed that all co-authors have no conflict of interests to declare. There have been no involvements that might raise the question of bias in the work reported or in the conclusions, implications or opinions stated. K.Y. and T.T. decided study design; M.W. did dietary education and calculated nutrition intakes; K.Y., T.T. and E.H. did data analysis. All authors contributed to write the paper and discussed the results.

\section{References}

1. Adolescent Health Committee, Canadian Paediatric Society (2004) Dieting in adolescence. Paediatr Child Health 9, 487-491.

2. Ministry of Education, Culture, Sports, Science and Technology (2006) School Health Examination Survey. Tokyo: Ministry of Education, Culture, Sports, Science and Technology.

3. Doak CM, Visscher TLS, Renders CM et al. (2006) The prevention of overweight and obesity in children and adolescents: a review of interventions and programs. Obes Rev 7, 111-136.

4. Stanfield PS \& Hui YH (2003) Nutrition and Diet Therapy: Self-Instructional Modules. Sudburry, MA: Jones \& Bartlett Publishers.

5. Willett WC (1990) Nutritional Epidemiology. New York: Oxford University Press.
6. Watanabe M, Yamaoka K, Adachi M et al. (2010) Validity and reproducibility of the FFQ (FFQW82) for dietary assessment in female adolescents. Public Health Nutr (Epublication ahead of print version).

7. Matthys C, De Henauw S, Bellemans M et al. (2007) Breakfast habits affect overall nutrient profiles in adolescents. Public Health Nutr 10, 413-421.

8. Chitra U \& Reddy CR (2007) The role of breakfast in nutrient intake of urban schoolchildren. Public Health Nutr 10, 55-58.

9. Sackett DL, Rosenberg WM, Gray JA et al. (1996) Evidence based medicine: what it is and what it isn't. BMJ 312, $71-72$.

10. Jansen W, Raat H, Joosten-van Zwanenburg E et al. (2008) A school-based intervention to reduce overweight and inactivity in children aged 6-12 years: study design of a randomized controlled trial. BMC Public Health 8, $257-265$.

11. Watanabe W, Yokotsuka M, Hiratsuka N et al. (2007) An approach to dietary education for Japanese urban schoolgirls - analysis of dietary consciousness, dietary behavior, dietary environment, and dietary intake. Bulletin of Universities and Institutes 806, 1-8.

12. Little RC, Milliken GA, Stroup WW et al. (1996) SAS System for Mixed Models, pp. 633. Cary, NC: SAS Institute Inc.

13. Campbell MK, Elbourne DR \& Altman DG (2004) CONSORT statement: extension to cluster randomized trials. BMJ 328, 702-708.

14. Cole TJ, Flegal KM, Nicholls D et al. (2007) Body mass index cut offs to define thinness in children and adolescents: international survey. BMJ 335, 194.

15. Cole TJ, Bellizzi MC, Flegal KM et al. (2000) Establishing a standard definition for child overweight and obesity worldwide: international survey. BMJ 320, 1240-1243.

16. Health Care Bureau, Ministry of Health and Welfare (2000) Promotion of the National Health Promotion Movement in the 21st Century (Healthy Japan 21). Tokyo: Ministry of Health and Welfare.

17. Ask A, Hernes S, Aarek I et al. (2006) Changes in dietary pattern in 15 year old adolescents following a 4 month dietary intervention with school breakfast - a pilot study. Nutr J 5, 33-38.

18. Larson NI, Sztainer DN, Hannan PJ et al. (2007) Family meals during adolescent are associated with higher diet quality and healthful meal patterns during young adulthood. J Am Diet Assoc 107, 1502-1510.

19. Fulkerson JA, Strauss J, Neumark-Sztainer D et al. (2007) Correlates of psychosocial well-being among overweight adolescents: the role of the family. J Consult Clin Psychol 75, 181-186. 